\title{
The Interpolative Berinde Weak Mapping Theorem in $\eta$-Cone Pentagonal Metric Space
}

\author{
Clement Boateng Ampadu
}

31 Carrolton Road, Boston, MA 02132-6303, USA

e-mail: drampadu@hotmail.com

\begin{abstract}
In this paper we introduce a concept of $\eta$-cone pentagonal metric space, which combines the notions of cone pentagonal metric space [1], and $\eta$-cone metric space 2]. Moreover, a variant of the interpolative Berinde weak mapping theorem obtained in 3 is proved in this setting.
\end{abstract}

\section{Introduction and Premilinaries}

Definition 1.1. 4] Let $E$ be a real Banach space with norm $\|\cdot\|$ and $P$ be a subset of $E$. Then $P$ is called a cone if and only if

(a) $P$ is closed, nonempty, and $P \neq\{\theta\}$, where $\theta$ is the zero vector in $E$;

(b) for any nonnegative real numbers $a$ and $b$, and $x, y \in P$, we have $a x+b y \in P$;

(c) for $x \in P$, if $-x \in P$, then $x=\theta$.

Definition 1.2. 4] Given a cone $P$ in a Banach space $E$, we define on $E$ a partial order $\preceq$ with respect to $P$ by

$$
x \preceq y \Longleftrightarrow y-x \in \operatorname{int}(P) .
$$

We shall write $x \prec y$ whenever $x \preceq y$ and $x \neq y$. While $x \ll y$ will stand for $y-x \in \operatorname{Int}(P)$, where $\operatorname{Int}(P)$ designates the interior of $P$.

Received: August 16, 2020; Accepted: September 19, 2020

2010 Mathematics Subject Classification: 47H10, 54H25.

Keywords and phrases: cone pentagonal metric space, fixed points, interpolative Berinde weak contraction mapping principle, ordered Banach space. 
Definition 1.3. 4] The cone $P$ is said to be normal if there is a real number $C>0$ such that for all $x, y \in E$, we have

$$
\theta \preceq x \preceq y \Longrightarrow\|x\| \leq C\|y\| .
$$

The least positive number satisfying the above inequality is called the normal constant of $P$. In particular, we will say that $P$ is a $K$-normal cone to indicate the fact that the normal constant is $K$.

Definition 1.4. [1] Let $X$ be a nonempty set. Suppose the mapping $d: X \times X \mapsto E$ satisfies

(a) $0 \leq d(x, y)$, for all $x, y \in X$ and $d(x, y)=0$ iff $x=y$;

(b) $d(x, y)=d(y, x)$ for all $x, y \in X$;

(c) $d(x, y) \leq d(x, z)+d(z, w)+d(w, u)+d(u, y)$ for all $x, y \in X$ and for all distinct points $z, w, u \in X-\{x, y\}$ [pentagonal property].

Then $d$ is called a cone pentagonal metric on $X$, and $(X, d)$ is called a cone pentagonal metric space.

Example 1.5. [5] Let $X=\{r, s, t, u, v\}, E=\mathbb{R}^{2}$, and $P=\{(x, y): x, y \geq 0\}$ be a cone in $E$. Define $d: X \times X \mapsto E$ as follows

$$
\begin{aligned}
d(x, x) & =0, \text { for all } x \in X, \\
d(r, s) & =d(s, r)=(4,8), \\
d(r, t) & =d(t, r)=d(t, u)=d(u, t)=d(s, t) \\
& =d(t, s)=d(s, u)=d(u, s)=d(r, u)=d(u, r)=(1,2), \\
d(r, v) & =d(v, r)=d(s, v)=d(v, s)=d(t, v)=d(v, t)=d(u, v)=d(v, u)=(3,6) .
\end{aligned}
$$

Then $(X, d)$ is a complete cone pentagonal metric space, but not a complete cone rectangular metric space, as it lacks the triangular property:

$$
\begin{aligned}
(4,8) & =d(r, s)>d(r, t)+d(t, u)+d(u, s) \\
& =(1,2)+(1,2)+(1,2)=(3,6)
\end{aligned}
$$

as $(4,8)-(3,6)=(1,2) \in P$. 
Definition 1.6. 2] Let $X$ be a nonempty set and $\eta: X \times X \mapsto[1, \infty)$ be a map. A function $d_{\eta}: X \times X \mapsto E$ will be called an $\eta$-cone metric on $X$ if

(a) $\theta \preceq d_{\eta}(x, y)$ for all $x \in X$ and $d_{\eta}(x, y)=\theta$ iff $x=y$;

(b) $d_{\eta}(x, y)=d_{\eta}(y, x)$ for all $x, y \in X$;

(c) $d_{\eta}(x, z) \preceq \eta(x, z)\left[d_{\eta}(x, y)+d_{\eta}(y, z)\right]$ for all $x, y, z \in X$.

Moreover the pair $\left(X, d_{\eta}\right)$ is called an $\eta$-cone metric space.

Remark 1.7. 2] If for all $x, y \in X$

(a) $\eta(x, y)=1$, then we obtain the definition of cone metric space [4].

(b) $\eta(x, y)=L$, where $L \geq 1$, then we obtain the definition of cone metric type space [6].

(c) $\eta(x, y)=C$, where $C \geq 1, E=\mathbb{R}$ and $P=[0, \infty)$, then we obtain the definition of metric type space [7].

Example 1.8. 2] Let $E=\mathbb{R}^{2}, P=\{(x, y) \in E: x, y \geq 0\} \subseteq \mathbb{R}^{2}$ and $X=\{1,2,3\}$. Let $\alpha \geq 0$ be a constant and define $\eta: X \times X \mapsto[1, \infty)$ and $d_{\eta}: X \times X \mapsto E$ by

$$
\begin{gathered}
\eta(x, y)=1+x+y, \\
d_{\eta}(1,1)=d_{\eta}(2,2)=d_{\eta}(3,3)=(0,0), \\
d_{\eta}(1,2)=d_{\eta}(2,1)=80(1, \alpha), \\
d_{\eta}(1,3)=d_{\eta}(3,1)=1000(1, \alpha), \\
d_{\eta}(2,3)=d_{\eta}(3,2)=600(1, \alpha) .
\end{gathered}
$$

Then $\left(X, d_{\eta}\right)$ is an $\eta$-cone metric space.

Now we introduce the following

Definition 1.9. Let $X$ be a nonempty set and $\eta: X \times X \mapsto[1, \infty)$ be a map. A function $d_{\eta}: X \times X \mapsto E$ will be called an $\eta$-cone pentagonal metric on $X$ if 
(a) $\theta \preceq d_{\eta}(x, y)$ for all $x, y \in X$ and $d_{\eta}(x, y)=\theta$ iff $x=y$;

(b) $d_{\eta}(x, y)=d_{\eta}(y, x)$ for all $x, y \in X$;

(c) $d_{\eta}(x, y) \leq \eta(x, y)\left[d_{\eta}(x, w)+d_{\eta}(w, z)+d_{\eta}(z, v)+d_{\eta}(v, y)\right]$ for all $x, y \in X$ and for all distinct points $w, z, v \in X-\{x, y\}$.

Moreover the pair $\left(X, d_{\eta}\right)$ will be called an $\eta$-cone pentagonal metric space.

Example 1.10. Let $X=\{r, s, t, u, v\}, E=\mathbb{R}^{2}$, and $P=\{(x, y): x, y \geq 0\}$ be a cone in $E$. Define $d_{\eta}: X \times X \mapsto E$ as follows

$$
\begin{aligned}
d_{\eta}(x, x) & =(0,0), \text { for all } x \in X, \\
d_{\eta}(r, s) & =d_{\eta}(s, r)=(4,8), \\
d_{\eta}(r, t) & =d_{\eta}(t, r)=d_{\eta}(t, u)=d_{\eta}(u, t)=d_{\eta}(s, t)=d_{\eta}(t, s)=d_{\eta}(s, u)=d_{\eta}(u, s) \\
& =d_{\eta}(r, u)=d_{\eta}(u, r)=(1,2), \\
d_{\eta}(r, v) & =d_{\eta}(v, r)=d_{\eta}(s, v)=d_{\eta}(v, s)=d_{\eta}(t, v)=d_{\eta}(v, t) \\
& =d_{\eta}(u, v)=d_{\eta}(v, u)=(3,6) .
\end{aligned}
$$

Also define $\eta: X \times X \mapsto[1, \infty)$ as follows

$$
\begin{aligned}
\eta(x, x) & =0, \text { for all } x \in X, \\
\eta(r, s) & =\eta(s, r)=\frac{1}{2} \\
\eta(r, t) & =\eta(t, r)=\eta(t, u)=\eta(u, t)=\eta(s, t)=\eta(t, s)=\eta(s, u)=\eta(u, s) \\
& =\eta(r, u)=\eta(u, r)=\frac{1}{11}, \\
\eta(r, v) & =\eta(v, r)=\eta(s, v)=\eta(v, s)=\eta(t, v)=\eta(v, t)=\eta(u, v) \\
& =\eta(v, u)=\frac{1}{3} .
\end{aligned}
$$

By definition of $d_{\eta}$, it is trivial to check Definition 1.9(a), and Definition 1.9(b). Now we check Definition 1.9(c). Obviously we have the following cases 
Case 1: $(x, x)$ for all $x \in X$

In this case it is enough to check

$$
d_{\eta}(r, r) \leq \eta(r, r)\left[d_{\eta}(r, s)+d_{\eta}(s, t)+d_{\eta}(t, u)+d_{\eta}(u, r)\right]
$$

Since $d_{\eta}(r, r)=(0,0)$ and $\eta(r, r)=0$, we have equality. In particular, we have

$$
\begin{aligned}
(0,0) & =d_{\eta}(r, r) \leq \eta(r, r)\left[d_{\eta}(r, s)+d_{\eta}(s, t)+d_{\eta}(t, u)+d_{\eta}(u, r)\right] \\
& =0\left[d_{\eta}(r, s)+d_{\eta}(s, t)+d_{\eta}(t, u)+d_{\eta}(u, r)\right]=(0,0) .
\end{aligned}
$$

Case 2: $(r, s)=(s, r)$

In this case it is enough to check

$$
d_{\eta}(r, s) \leq \eta(r, s)\left[d_{\eta}(r, t)+d_{\eta}(t, u)+d_{\eta}(u, v)+d_{\eta}(v, s)\right] .
$$

In particular, we have

$$
\begin{aligned}
(4,8) & =d_{\eta}(r, s) \\
& \leq \eta(r, s)\left[d_{\eta}(r, t)+d_{\eta}(t, u)+d_{\eta}(u, v)+d_{\eta}(v, s)\right] \\
& =\frac{1}{2}[(1,2)+(1,2)+(3,6)+(3,6)] \\
& =\frac{1}{2}(8,16)=(4,8) .
\end{aligned}
$$

$\underline{\text { Case 3: }(r, t)=(t, r)=(t, u)=(u, t)=(s, t)=(t, s)=(s, u)=(u, s)=(r, u)=(u, r)}$

In this case it is enough to check

$$
d_{\eta}(r, t) \leq \eta(r, t)\left[d_{\eta}(r, s)+d_{\eta}(s, u)+d_{\eta}(u, v)+d_{\eta}(v, t)\right]
$$


In particular, we have

$$
\begin{aligned}
(1,2) & =d_{\eta}(r, t) \\
& \leq \eta(r, t)\left[d_{\eta}(r, s)+d_{\eta}(s, u)+d_{\eta}(u, v)+d_{\eta}(v, t)\right] \\
& =\frac{1}{11}\left[(4,8)+(1,2)+(3,6)+(3,6)=\frac{1}{11}(11,22)=(1,2) .\right.
\end{aligned}
$$

Case 4: $(r, v)=(v, r)=(s, v)=(v, s)=(t, v)=(v, t)=(u, v)=(v, u)$

In this case it is enough to check that

$$
d_{\eta}(r, v) \leq \eta(r, v)\left[d_{\eta}(r, s)+d_{\eta}(s, t)+d_{\eta}(t, u)+d_{\eta}(u, v)\right] .
$$

In particular, we have

$$
\begin{aligned}
(3,6) & =d_{\eta}(r, v) \\
& \leq \eta(r, v)\left[d_{\eta}(r, s)+d_{\eta}(s, t)+d_{\eta}(t, u)+d_{\eta}(u, v)\right] \\
& =\frac{1}{3}[(4,8)+(1,2)+(1,2)+(3,6)]=\frac{1}{3}(9,18)=(3,6) .
\end{aligned}
$$

It follows that $\left(X, d_{\eta}\right)$ is an $\eta$-cone pentagonal metric space. Note that $\left(X, d_{\eta}\right)$ is not an $\eta$-cone rectangular metric space, as it lacks Definition 1.9(c) [8]. In particular,

$$
\begin{aligned}
(4,8) & =d_{\eta}(r, s)>\eta(r, s)\left[d_{\eta}(r, r)+d_{\eta}(r, t)+d_{\eta}(t, s)\right] \\
& =\frac{1}{2}[(0,0)+(1,2)+(1,2)] \\
& =\frac{1}{2}(2,4)=(1,2)
\end{aligned}
$$

as $(4,8)-(1,2)=(3,6) \in P$.

Definition 1.11. Let $\left(X, d_{\eta}\right)$ be a $\eta$-cone pentagonal metric space. Let $\left\{x_{n}\right\}$ be a sequence in $\left(X, d_{\eta}\right)$ and $x \in X$. If for every $c \in E$ with $0 \ll c$, there exists $n_{0} \in \mathbb{N}$ such that for all $n>n_{0}, d_{\eta}\left(x_{n}, c\right) \ll c$, then we say $\left\{x_{n}\right\}$ is convergent, and $\left\{x_{n}\right\}$ converges to $x$. Moreover, $x$ will be called the limit of $\left\{x_{n}\right\}$. We sometimes write $\lim _{n \rightarrow \infty} x_{n}=x$, or $x_{n} \rightarrow x$ as $n \rightarrow \infty$. 
Definition 1.12. Let $\left(X, d_{\eta}\right)$ be a $\eta$-cone pentagonal metric space. If for every $c \in E$, with $0 \ll c$, there exists $n_{0} \in \mathbb{N}$ such that for all $n>n_{0}, d_{\eta}\left(x_{n}, x\right) \ll c$, then we say $\left\{x_{n}\right\}$ is a Cauchy sequence in $\left(X, d_{\eta}\right)$.

Definition 1.13. Let $\left(X, d_{\eta}\right)$ be a $\eta$-cone pentagonal metric space. If every Cauchy sequence is convergent in $\left(X, d_{\eta}\right)$, then $\left(X, d_{\eta}\right)$ will be called a complete $\eta$-cone pentagonal metric space.

Notation 1.14. [1] Let $P$ be a cone as defined in this paper. $\Phi$ will denote the set of all nondcreasing continuous functions $\varphi: P \mapsto P$ satisfying

(a) $0<\varphi(t)<t$ for all $t \in P \backslash\{0\}$;

(b) the series $\sum_{n \geq 0} \varphi^{n}(t)$ converges for all $t \in P \backslash\{0\}$.

Note that from $(a)$, we have $\varphi(0)=0$, and from $(b)$ we have $\lim _{n \rightarrow \infty} \varphi^{n}(t)=0$ for all $t \in P \backslash\{0\}$.

Definition 1.15. Let $\left(X, d_{\eta}\right)$ be a $\eta$-cone pentagonal metric space. A map $T: X \mapsto$ $X$ will be called an (alternate) Interpolative Berinde Weak operator if it satisfies

$$
d_{\eta}(T x, T y) \leq \lambda d_{\eta}(x, y)^{\frac{1}{2}} d_{\eta}(x, T x)^{\frac{1}{2}},
$$

where $\lambda \in(0,1)$, for all $x, y \in X, x, y \notin F i x(T)$, where $\operatorname{Fix}(T)=\{x \in X: T x=x\}$.

\section{Main Results}

Our main result is as follows

Theorem 2.1. Let $\left(X, d_{\eta}\right)$ be a $\eta$-cone pentagonal metric space. Suppose $T: X \mapsto X$ satisfies

$$
d_{\eta}(T x, T y) \leq \varphi\left(\frac{1}{\sqrt{2}} d_{\eta}(x, y)^{\frac{1}{2}} d_{\eta}(x, T x)^{\frac{1}{2}}\right)
$$

for all $x, y \in X, x, y \notin F i x(T)$, and $\varphi \in \Phi$. If $\left(X, d_{\eta}\right)$ is complete and

$$
\lim _{n, m \rightarrow \infty} \eta\left(x_{0}, x_{m}\right)
$$

exists and is finite, then the fixed point of $T$ exists. 
Proof. Let $x_{0}$ be an arbitrary point in $X$. Define a sequence $\left\{x_{n}\right\}$ in $X$ by $x_{n+1}=$ $T x_{n}$ for all $n=0,1,2, \cdots$. Assume that $x_{n} \neq x_{n+1}$ for all $n \in \mathbb{N}$. Now observe from the inequality of the theorem, we have

$$
\begin{aligned}
d_{\eta}\left(x_{n}, x_{n+1}\right) & =d_{\eta}\left(T x_{n-1}, T x_{n}\right) \\
& \leq \varphi\left(\frac{1}{\sqrt{2}} d_{\eta}\left(x_{n-1}, x_{n}\right)^{\frac{1}{2}} d_{\eta}\left(x_{n-1}, T x_{n-1}\right)^{\frac{1}{2}}\right) \\
& =\varphi\left(\frac{1}{\sqrt{2}} d_{\eta}\left(x_{n-1}, x_{n}\right)^{\frac{1}{2}} d_{\eta}\left(x_{n-1}, x_{n}\right)^{\frac{1}{2}}\right) \\
& <\varphi\left(d_{\eta}\left(x_{n-1}, x_{n}\right)\right) \\
& =\varphi\left(d_{\eta}\left(T x_{n-2}, T x_{n-1}\right)\right) \\
& \leq \varphi\left(\varphi\left(d_{\eta}\left(x_{n-2}, x_{n-1}\right)\right)\right) \\
& =\varphi^{2}\left(d_{\eta}\left(x_{n-2}, x_{n-1}\right)\right) \\
& \vdots \\
& \leq \varphi^{n}\left(d_{\eta}\left(x_{0}, x_{1}\right)\right) .
\end{aligned}
$$

Now observe we have the following

$$
\begin{aligned}
d_{\eta}\left(x_{n}, x_{n+2}\right) & =d_{\eta}\left(T x_{n-1}, T x_{n+1}\right) \\
& \leq \varphi\left(\frac{1}{\sqrt{2}} d_{\eta}\left(x_{n-1}, x_{n+1}\right)^{\frac{1}{2}} d_{\eta}\left(x_{n-1}, T x_{n-1}\right)^{\frac{1}{2}}\right) \\
& =\varphi\left(\frac{1}{\sqrt{2}} d_{\eta}\left(x_{n-1}, x_{n+1}\right)^{\frac{1}{2}} d_{\eta}\left(x_{n-1}, x_{n}\right)^{\frac{1}{2}}\right) \\
& \leq \varphi\left(\frac{1}{\sqrt{2}} d_{\eta}\left(x_{n-1}, x_{n+1}\right)^{\frac{1}{2}} 2^{\frac{1}{2}} d_{\eta}\left(x_{n-1}, x_{n+1}\right)^{\frac{1}{2}}\right) \\
& =\varphi\left(d_{\eta}\left(x_{n-1}, x_{n+1}\right)\right) \\
& =\varphi\left(d_{\eta}\left(T x_{n-2}, T x_{n}\right)\right)
\end{aligned}
$$




$$
\begin{aligned}
& \leq \varphi\left(\varphi\left(d_{\eta}\left(x_{n-2}, x_{n}\right)\right)\right) \\
& =\varphi^{2}\left(d_{\eta}\left(x_{n-2}, x_{n}\right)\right) \\
& \vdots \\
& \leq \varphi^{n}\left(d_{\eta}\left(x_{0}, x_{2}\right)\right) .
\end{aligned}
$$

Further we deduce the following

$$
\begin{aligned}
d_{\eta}\left(x_{n}, x_{n+3}\right) & =d_{\eta}\left(T x_{n-1}, T x_{n+2}\right) \\
& \leq \varphi\left(\frac{1}{\sqrt{2}} d_{\eta}\left(x_{n-1}, x_{n+2}\right)^{\frac{1}{2}} d_{\eta}\left(x_{n-1}, T x_{n-1}\right)^{\frac{1}{2}}\right) \\
& =\varphi\left(\frac{1}{\sqrt{2}} d_{\eta}\left(x_{n-1}, x_{n+2}\right)^{\frac{1}{2}} d_{\eta}\left(x_{n-1}, x_{n}\right)^{\frac{1}{2}}\right) \\
& \leq \varphi\left(\frac{1}{\sqrt{2}} d_{\eta}\left(x_{n-1}, x_{n+2}\right)^{\frac{1}{2}} 2^{\frac{1}{2}} d_{\eta}\left(x_{n-1}, x_{n+2}\right)^{\frac{1}{2}}\right) \\
& =\varphi\left(d_{\eta}\left(x_{n-1}, x_{n+2}\right)\right) \\
& =\varphi\left(d_{\eta}\left(T x_{n-2}, T x_{n+1}\right)\right) \\
& \leq \varphi\left(\varphi\left(d_{\eta}\left(x_{n-2}, x_{n+1}\right)\right)\right) \\
& =\varphi^{2}\left(d_{\eta}\left(x_{n-2}, x_{n+1}\right)\right) \\
& \vdots \\
& \leq \varphi^{n}\left(d_{\eta}\left(x_{0}, x_{3}\right)\right) .
\end{aligned}
$$

Now for $k=1,2,3, \cdots$ we have

$$
d_{\eta}\left(x_{n}, x_{n+3 k+1}\right) \leq \varphi^{n}\left(d_{\eta}\left(x_{0}, x_{3 k+1}\right)\right)
$$




$$
\begin{aligned}
& d_{\eta}\left(x_{n}, x_{n+3 k+2}\right) \leq \varphi^{n}\left(d_{\eta}\left(x_{0}, x_{3 k+2}\right)\right) \\
& d_{\eta}\left(x_{n}, x_{n+3 k+3}\right) \leq \varphi^{n}\left(d_{\eta}\left(x_{0}, x_{3 k+3}\right)\right) .
\end{aligned}
$$

Since $d_{\eta}\left(x_{n}, x_{n+1}\right) \leq \varphi^{n}\left(d_{\eta}\left(x_{0}, x_{1}\right)\right)$, we deduce the following using the $\eta$-cone pentagonal property

$$
\begin{aligned}
d \eta\left(x_{0}, x_{4}\right) & \leq \eta\left(x_{0}, x_{4}\right)\left[d \eta\left(x_{0}, x_{1}\right)+d \eta\left(x_{1}, x_{2}\right)+d \eta\left(x_{2}, x_{3}\right)+d \eta\left(x_{3}, x_{4}\right)\right] \\
& \leq \eta\left(x_{0}, x_{4}\right)\left[d \eta\left(x_{0}, x_{1}\right)+\varphi\left(d_{\eta}\left(x_{0}, x_{1}\right)\right)+\varphi^{2}\left(d_{\eta}\left(x_{0}, x_{1}\right)\right)+\varphi^{3}\left(d_{\eta}\left(x_{0}, x_{1}\right)\right)\right] \\
& \leq \eta\left(x_{0}, x_{4}\right)\left[\sum_{i=0}^{3} \varphi^{i}\left(d_{\eta}\left(x_{0}, x_{1}\right)\right)\right] .
\end{aligned}
$$

Similarly, we can show the following

$$
d \eta\left(x_{0}, x_{7}\right) \leq \eta\left(x_{0}, x_{7}\right)\left[\sum_{i=0}^{6} \varphi^{i}\left(d_{\eta}\left(x_{0}, x_{1}\right)\right)\right] .
$$

By induction for $k=1,2,3, \cdots$, we have

$$
d \eta\left(x_{0}, x_{3 k+1}\right) \leq \eta\left(x_{0}, x_{3 k+1}\right)\left[\sum_{i=0}^{3 k} \varphi^{i}\left(d_{\eta}\left(x_{0}, x_{1}\right)\right)\right] .
$$

Since $d_{\eta}\left(x_{n}, x_{n+1}\right) \leq \varphi^{n}\left(d_{\eta}\left(x_{0}, x_{1}\right)\right)$ and $d_{\eta}\left(x_{n}, x_{n+2}\right) \leq \varphi^{n}\left(d_{\eta}\left(x_{0}, x_{2}\right)\right)$, we deduce the following using the $\eta$-cone pentagonal property

$$
\begin{aligned}
d \eta\left(x_{0}, x_{5}\right) & \leq \eta\left(x_{0}, x_{5}\right)\left[d \eta\left(x_{0}, x_{1}\right)+d \eta\left(x_{1}, x_{2}\right)+d \eta\left(x_{2}, x_{3}\right)+d \eta\left(x_{3}, x_{5}\right)\right] \\
& \leq \eta\left(x_{0}, x_{5}\right)\left[d \eta\left(x_{0}, x_{1}\right)+\varphi\left(d_{\eta}\left(x_{0}, x_{1}\right)\right)+\varphi^{2}\left(d_{\eta}\left(x_{0}, x_{1}\right)\right)+\varphi^{3}\left(d_{\eta}\left(x_{0}, x_{2}\right)\right)\right] \\
& \leq \eta\left(x_{0}, x_{5}\right)\left[\sum_{i=0}^{2} \varphi^{i}\left(d_{\eta}\left(x_{0}, x_{1}\right)\right)+\varphi^{3}\left(d_{\eta}\left(x_{0}, x_{2}\right)\right)\right] .
\end{aligned}
$$


Similarly, we can show the following

$$
d \eta\left(x_{0}, x_{8}\right) \leq \eta\left(x_{0}, x_{8}\right)\left[\sum_{i=0}^{5} \varphi^{i}\left(d_{\eta}\left(x_{0}, x_{1}\right)\right)+\varphi^{6}\left(d_{\eta}\left(x_{0}, x_{2}\right)\right)\right] .
$$

By induction for $k=1,2,3, \cdots$, we have

$$
d \eta\left(x_{0}, x_{3 k+2}\right) \leq \eta\left(x_{0}, x_{3 k+2}\right)\left[\sum_{i=0}^{3 k-1} \varphi^{i}\left(d_{\eta}\left(x_{0}, x_{1}\right)\right)+\varphi^{3 k}\left(d_{\eta}\left(x_{0}, x_{2}\right)\right)\right] .
$$

Since $d_{\eta}\left(x_{n}, x_{n+1}\right) \leq \varphi^{n}\left(d_{\eta}\left(x_{0}, x_{1}\right)\right)$ and $d_{\eta}\left(x_{n}, x_{n+3}\right) \leq \varphi^{n}\left(d_{\eta}\left(x_{0}, x_{3}\right)\right)$, we deduce the following using the $\eta$-cone pentagonal property

$$
\begin{aligned}
d \eta\left(x_{0}, x_{6}\right) & \leq \eta\left(x_{0}, x_{6}\right)\left[d \eta\left(x_{0}, x_{1}\right)+d \eta\left(x_{1}, x_{2}\right)+d \eta\left(x_{2}, x_{3}\right)+d \eta\left(x_{3}, x_{6}\right)\right] \\
& \leq \eta\left(x_{0}, x_{6}\right)\left[d \eta\left(x_{0}, x_{1}\right)+\varphi\left(d_{\eta}\left(x_{0}, x_{1}\right)\right)+\varphi^{2}\left(d_{\eta}\left(x_{0}, x_{1}\right)\right)+\varphi^{3}\left(d_{\eta}\left(x_{0}, x_{3}\right)\right)\right] \\
& \leq \eta\left(x_{0}, x_{6}\right)\left[\sum_{i=0}^{2} \varphi^{i}\left(d_{\eta}\left(x_{0}, x_{1}\right)\right)+\varphi^{3}\left(d_{\eta}\left(x_{0}, x_{3}\right)\right)\right] .
\end{aligned}
$$

Similarly, we can show the following

$$
d \eta\left(x_{0}, x_{9}\right) \leq \eta\left(x_{0}, x_{9}\right)\left[\sum_{i=0}^{5} \varphi^{i}\left(d_{\eta}\left(x_{0}, x_{1}\right)\right)+\varphi^{6}\left(d_{\eta}\left(x_{0}, x_{3}\right)\right)\right] .
$$

By induction for $k=1,2,3, \cdots$, we have

$$
d \eta\left(x_{0}, x_{3 k+3}\right) \leq \eta\left(x_{0}, x_{3 k+3}\right)\left[\sum_{i=0}^{3 k-1} \varphi^{i}\left(d_{\eta}\left(x_{0}, x_{1}\right)\right)+\varphi^{3 k}\left(d_{\eta}\left(x_{0}, x_{3}\right)\right)\right] .
$$

Since

$$
d_{\eta}\left(x_{n}, x_{n+3 k+1}\right) \leq \varphi^{n}\left(d_{\eta}\left(x_{0}, x_{3 k+1}\right)\right)
$$

and

$$
d \eta\left(x_{0}, x_{3 k+1}\right) \leq \eta\left(x_{0}, x_{3 k+1}\right)\left[\sum_{i=0}^{3 k} \varphi^{i}\left(d_{\eta}\left(x_{0}, x_{1}\right)\right)\right],
$$


we deduce the following

$$
\begin{aligned}
d_{\eta}\left(x_{n}, x_{n+3 k+1}\right) & \leq \varphi^{n}\left(d_{\eta}\left(x_{0}, x_{3 k+1}\right)\right) \\
& \leq \varphi^{n}\left(\eta\left(x_{0}, x_{3 k+1}\right)\left[\sum_{i=0}^{3 k} \varphi^{i}\left(d_{\eta}\left(x_{0}, x_{1}\right)\right)\right]\right) \\
& \leq \varphi^{n}\left(\eta\left(x_{0}, x_{3 k+1}\right)\left[\sum_{i=0}^{3 k} \varphi^{i}\left(d_{\eta}\left(x_{0}, x_{1}\right)+d_{\eta}\left(x_{0}, x_{2}\right)+d_{\eta}\left(x_{0}, x_{3}\right)\right)\right]\right) \\
& \leq \varphi^{n}\left(\eta\left(x_{0}, x_{3 k+1}\right)\left[\sum_{i=0}^{\infty} \varphi^{i}\left(d_{\eta}\left(x_{0}, x_{1}\right)+d_{\eta}\left(x_{0}, x_{2}\right)+d_{\eta}\left(x_{0}, x_{3}\right)\right)\right]\right) .
\end{aligned}
$$

Since $d_{\eta}\left(x_{n}, x_{n+3 k+2}\right) \leq \varphi^{n}\left(d_{\eta}\left(x_{0}, x_{3 k+2}\right)\right)$ and

$$
d \eta\left(x_{0}, x_{3 k+2}\right) \leq \eta\left(x_{0}, x_{3 k+2}\right)\left[\sum_{i=0}^{3 k-1} \varphi^{i}\left(d_{\eta}\left(x_{0}, x_{1}\right)\right)+\varphi^{3 k}\left(d_{\eta}\left(x_{0}, x_{2}\right)\right)\right],
$$

we deduce the following

$$
\begin{aligned}
d_{\eta}\left(x_{n}, x_{n+3 k+2}\right) & \leq \varphi^{n}\left(d_{\eta}\left(x_{0}, x_{3 k+2}\right)\right) \\
& \leq \varphi^{n}\left(\eta\left(x_{0}, x_{3 k+2}\right)\left[\sum_{i=0}^{3 k-1} \varphi^{i}\left(d_{\eta}\left(x_{0}, x_{1}\right)\right)+\varphi^{3 k}\left(d_{\eta}\left(x_{0}, x_{2}\right)\right)\right]\right) \\
& \leq \varphi^{n}\left(\eta ( x _ { 0 } , x _ { 3 k + 2 } ) \left[\sum_{i=0}^{3 k-1} \varphi^{i}\left(d_{\eta}\left(x_{0}, x_{1}\right)+d_{\eta}\left(x_{0}, x_{2}\right)+d_{\eta}\left(x_{0}, x_{3}\right)\right)\right.\right. \\
& \left.\left.+\varphi^{3 k}\left(d_{\eta}\left(x_{0}, x_{1}\right)+d_{\eta}\left(x_{0}, x_{2}\right)+d_{\eta}\left(x_{0}, x_{3}\right)\right)\right]\right) \\
& \leq \varphi^{n}\left(\eta\left(x_{0}, x_{3 k+2}\right)\left[\sum_{i=0}^{3 k} \varphi^{i}\left(d_{\eta}\left(x_{0}, x_{1}\right)+d_{\eta}\left(x_{0}, x_{2}\right)+d_{\eta}\left(x_{0}, x_{3}\right)\right)\right]\right) \\
& \leq \varphi^{n}\left(\eta\left(x_{0}, x_{3 k+2}\right)\left[\sum_{i=0}^{\infty} \varphi^{i}\left(d_{\eta}\left(x_{0}, x_{1}\right)+d_{\eta}\left(x_{0}, x_{2}\right)+d_{\eta}\left(x_{0}, x_{3}\right)\right)\right]\right) .
\end{aligned}
$$


Since

$$
d_{\eta}\left(x_{n}, x_{n+3 k+3}\right) \leq \varphi^{n}\left(d_{\eta}\left(x_{0}, x_{3 k+3}\right)\right)
$$

and

$$
d \eta\left(x_{0}, x_{3 k+3}\right) \leq \eta\left(x_{0}, x_{3 k+3}\right)\left[\sum_{i=0}^{3 k-1} \varphi^{i}\left(d_{\eta}\left(x_{0}, x_{1}\right)\right)+\varphi^{3 k}\left(d_{\eta}\left(x_{0}, x_{3}\right)\right)\right],
$$

for $k=1,2,3, \cdots$ we can show the following

$d_{\eta}\left(x_{n}, x_{n+3 k+3}\right) \leq \varphi^{n}\left(\eta\left(x_{0}, x_{3 k+3}\right)\left[\sum_{i=0}^{\infty} \varphi^{i}\left(d_{\eta}\left(x_{0}, x_{1}\right)+d_{\eta}\left(x_{0}, x_{2}\right)+d_{\eta}\left(x_{0}, x_{3}\right)\right)\right]\right)$.

Consequently it follows for each $m$, we have the following

$$
d_{\eta}\left(x_{n}, x_{n+m}\right) \leq \varphi^{n}\left(\eta\left(x_{0}, x_{m}\right)\left[\sum_{i=0}^{\infty} \varphi^{i}\left(d_{\eta}\left(x_{0}, x_{1}\right)+d_{\eta}\left(x_{0}, x_{2}\right)+d_{\eta}\left(x_{0}, x_{3}\right)\right)\right]\right) .
$$

Since $\sum_{i=0}^{\infty} \varphi^{i}\left(d_{\eta}\left(x_{0}, x_{1}\right)+d_{\eta}\left(x_{0}, x_{2}\right)+d_{\eta}\left(x_{0}, x_{3}\right)\right)$ is convergent, where

$$
d_{\eta}\left(x_{0}, x_{1}\right)+d_{\eta}\left(x_{0}, x_{2}\right)+d_{\eta}\left(x_{0}, x_{3}\right) \in P \backslash\{0\}
$$

$P$ is closed, then $\sum_{i=0}^{\infty} \varphi^{i}\left(d_{\eta}\left(x_{0}, x_{1}\right)+d_{\eta}\left(x_{0}, x_{2}\right)+d_{\eta}\left(x_{0}, x_{3}\right)\right) \in P \backslash\{0\}$. Since $\lim _{n, m \rightarrow \infty} \eta\left(x_{0}, x_{m}\right)$ exists and is finite, then

$$
0=\lim _{n, m \rightarrow \infty} \varphi^{n}\left(\eta\left(x_{0}, x_{m}\right)\left[\sum_{i=0}^{\infty} \varphi^{i}\left(d_{\eta}\left(x_{0}, x_{1}\right)+d_{\eta}\left(x_{0}, x_{2}\right)+d_{\eta}\left(x_{0}, x_{3}\right)\right)\right]\right) .
$$

So given $0 \ll c$, we can find natural number $N_{1}$ such that

$$
\varphi^{n}\left(\eta\left(x_{0}, x_{m}\right)\left[\sum_{i=0}^{\infty} \varphi^{i}\left(d_{\eta}\left(x_{0}, x_{1}\right)+d_{\eta}\left(x_{0}, x_{2}\right)+d_{\eta}\left(x_{0}, x_{3}\right)\right)\right]\right) \ll c
$$

for all $n \geq N_{1}$. Consequently,

$$
d_{\eta}\left(x_{n}, x_{n+m}\right) \ll c \text { for all } n \geq N_{1} .
$$


Therefore $\left\{x_{n}\right\}$ is a Cauchy sequence in $X$. Since $X$ is complete, there is a point $z \in X$ such that

$$
\lim _{n \rightarrow \infty} x_{n}=\lim _{n \rightarrow \infty} T x_{n-1}=z .
$$

Finally we show existence of the fixed point, that is, $T z=z$. Since $0 \ll c$, we can choose natural numbers $N_{2}, N_{3}, N_{4}$ such that $d_{\eta}\left(z, x_{n}\right) \ll \frac{c}{4 \eta(T z, z)}$, for all $n \geq N_{2}$, $d_{\eta}\left(x_{n+1}, x_{n}\right) \ll \frac{c}{4 \eta(T z, z)}$, for all $n \geq N_{3}$, and $d_{\eta}\left(x_{n-1}, z\right) \ll \frac{c}{4 \eta(T z, z)}$, for all $n \geq N_{4}$. Since $x_{n} \neq x_{m}$ for $n \neq m$, therefore by $\eta$-cone pentagonal property, we deduce the following

$$
\begin{aligned}
d_{\eta}(T z, z) & \leq \eta(T z, z)\left[d_{\eta}\left(T z, T x_{n}\right)+d_{\eta}\left(T x_{n}, T x_{n-1}\right)+d_{\eta}\left(T x_{n-1}, T x_{n-2}\right)+d_{\eta}\left(T x_{n-2}, z\right)\right] \\
& \leq \eta(T z, z)\left[d_{\eta}\left(T z, T x_{n}\right)+d_{\eta}\left(x_{n+1}, x_{n}\right)+d_{\eta}\left(x_{n}, x_{n-1}\right)+d_{\eta}\left(x_{n-1}, z\right)\right] .
\end{aligned}
$$

By the inequality of the theorem, we have

$$
\begin{aligned}
d\left(T z, T x_{n}\right) & \leq \varphi\left(\frac{1}{\sqrt{2}} d_{\eta}\left(z, x_{n}\right)^{\frac{1}{2}} d_{\eta}(z, T z)^{\frac{1}{2}}\right) \\
& \leq \varphi\left(\frac{1}{\sqrt{2}} d_{\eta}\left(z, x_{n}\right)^{\frac{1}{2}}\left(d_{\eta}\left(z, x_{n}\right)+d_{\eta}\left(x_{n}, T z\right)\right)^{\frac{1}{2}}\right) \\
& \leq \varphi\left(\frac{1}{\sqrt{2}} d_{\eta}\left(z, x_{n}\right)^{\frac{1}{2}}\left(2 d_{\eta}\left(z, x_{n}\right)\right)^{\frac{1}{2}}\right) \\
& \leq \varphi\left(d_{\eta}\left(z, x_{n}\right)\right) \\
& <d_{\eta}\left(z, x_{n}\right) .
\end{aligned}
$$

Thus

$$
\begin{aligned}
d_{\eta}(T z, z) & \leq \eta(T z, z)\left[d_{\eta}\left(T z, T x_{n}\right)+d_{\eta}\left(T x_{n}, T x_{n-1}\right)+d_{\eta}\left(T x_{n-1}, T x_{n-2}\right)+d_{\eta}\left(T x_{n-2}, z\right)\right] \\
& \leq \eta(T z, z)\left[d_{\eta}\left(T z, T x_{n}\right)+d_{\eta}\left(x_{n+1}, x_{n}\right)+d_{\eta}\left(x_{n}, x_{n-1}\right)+d_{\eta}\left(x_{n-1}, z\right)\right] \\
& \leq \eta(T z, z)\left[d_{\eta}\left(z, x_{n}\right)+d_{\eta}\left(x_{n+1}, x_{n}\right)+d_{\eta}\left(x_{n}, x_{n-1}\right)+d_{\eta}\left(x_{n-1}, z\right)\right] .
\end{aligned}
$$

Hence

$$
d_{\eta}(T z, z) \ll \eta(T z, z)\left[4 \cdot \frac{c}{4 \eta(T z, z)}\right] \ll c
$$


for all $n \geq N$, where $N:=\max \left\{N_{2}, N_{3}, N_{4}\right\}$. Since $c$ is arbitrary, we have $d_{\eta}(T z, z) \ll \frac{c}{m}$ for all $m \in \mathbb{N}$. Since $\frac{c}{m} \rightarrow 0$ as $m \rightarrow \infty$, we conclude that $\frac{c}{m}-d_{\eta}(T z, z) \rightarrow-d_{\eta}(T z, z)$ as $m \rightarrow \infty$. Since $P$ is closed, $-d_{\eta}(T z, z) \in P$. Hence $d_{\eta}(T z, z) \in P \cap(-P)$. By definition of cone, we have $d_{\eta}(T z, z)=0$. So the fixed point exists, and the proof is finished.

\section{Open Problems}

To conclude this paper, we suggest some unsolved problems

Conjecture 3.1. Let $\left(X, d_{\eta}\right)$ be a $\eta$-cone pentagonal metric space. Suppose $T$ : $X \mapsto X$ satisfies the following for some positive integer $m$

$$
d_{\eta}\left(T^{m} x, T^{m} y\right) \leq \varphi\left(\frac{1}{\sqrt{2}} d_{\eta}(x, y)^{\frac{1}{2}} d_{\eta}\left(x, T^{m} x\right)^{\frac{1}{2}}\right)
$$

for all $x, y \in X, x, y \notin F i x(T)$, and $\varphi \in \Phi$. If $\left(X, d_{\eta}\right)$ is complete and

$$
\lim _{n, m \rightarrow \infty} \eta\left(x_{0}, x_{m}\right)
$$

exists and is finite, then the fixed point of $T$ exists.

Conjecture 3.2. Let $\left(X, d_{\eta}\right)$ be a $\eta$-cone pentagonal metric space. Suppose $T$ : $X \mapsto X$ satisfies the following

$$
d_{\eta}(T x, T y) \leq \lambda d_{\eta}(x, y)^{\frac{1}{2}} d_{\eta}(x, T x)^{\frac{1}{2}}
$$

for all $x, y \in X, x, y \notin F i x(T)$, and $\lambda \in(0,1)$. If $\left(X, d_{\eta}\right)$ is complete and

$$
\lim _{n, m \rightarrow \infty} \eta\left(x_{0}, x_{m}\right)
$$

exists and is finite, then the fixed point of $T$ exists.

Conjecture 3.3. Theorem 2.1 holds in $\eta$-cone rectangular metric space [8]. 


\section{References}

[1] Abba Auwalu, Banach fixed point theorem in a cone pentagonal metric spaces, Journal of Advanced Studies in Topology 7(2) (2016), 60-67. https://doi.org/10.20454/jast.2016.1019

[2] Yaé Ulrich Gaba, $\eta$-metric structures, 2017. arXiv:1709.07690 [math.GN]

[3] Clement Boateng Ampadu, Some fixed point theory results for the interpolative Berinde weak operator, Earthline Journal of Mathematical Sciences 4(2) (2020), 253-271. https://doi.org/10.34198/ejms.4220.253271

[4] L.-G. Huang and X. Zhang, Cone metric spaces and fixed point theorems of contractive mappings, Journal of Mathematical Analysis and Applications 332(2) (2007), 1468-1476. https://doi.org/10.1016/j.jmaa.2005.03.087

[5] Abba Auwalu and Evren Hıçal, Common fixed points of two maps in cone pentagonal metric spaces, Global Journal of Pure and Applied Mathematics 12(3) (2016), 2423-2435.

[6] A.S. Cvetković, M.P. Stanić, S. Dimitrijević and S. Simić, Common fixed point theorems for four mappings on cone metric type space, Fixed Point Theory Appl. 2011, Art. ID 589725, 15 pp. https://doi.org/10.1155/2011/589725

[7] M.A. Khamsi, Remarks on cone metric spaces and fixed point theorems of contractive mappings, Fixed Point Theory and Application 2010, Art. ID 315398, 7 pp. https://doi.org/10.1155/2010/315398

[8] Clement Boateng Ampadu, Banach contraction mapping theorem in $\eta$-cone rectangular metric space, Fundamental Journal of Mathematics and Mathematical Sciences 13(1) (2020), 23-34.

This is an open access article distributed under the terms of the Creative Commons Attribution License (http://creativecommons.org/licenses/by/4.0/), which permits unrestricted, use, distribution and reproduction in any medium, or format for any purpose, even commercially provided the work is properly cited. 